\title{
VLT-UVES analysis of 5 giants in 47 Tucanae ${ }^{\star \star \star \star}$
}

\author{
A. Alves-Brito ${ }^{1}$, B. Barbuy ${ }^{1}$, S. Ortolani ${ }^{2}$, Y. Momany $^{2}$, V. Hill ${ }^{3}$, M. Zoccali ${ }^{4}, 5$, A. Renzini ${ }^{6}$, \\ D. Minniti ${ }^{4}$, L. Pasquini ${ }^{6}$, E. Bica ${ }^{7}$, and R. M. Rich ${ }^{8}$
}

1 Universidade de São Paulo, IAG, Rua do Matão 1226, Cidade Universitária, São Paulo 05508-900, Brazil e-mail: [abrito; barbuy] @astro.iag.usp.br

2 Università di Padova, Dipartimento di Astronomia, Vicolo dell'Osservatorio 2, 35122 Padova, Italy e-mail: ortolani@pd.astro.it

${ }^{3}$ Observatoire de Paris-Meudon, 92195 Meudon Cedex, France e-mail: Vanessa.Hill@obspm.fr

${ }^{4}$ Universidad Catolica de Chile, Department of Astronomy \& Astrophysics, Casilla 306, Santiago 22, Chile e-mail: [dante;mzoccali]@astro.puc.cl

5 Princeton University Observatory, Peyton Hall, Princeton, NJ 08544, USA

${ }^{6}$ European Southern Observatory, Karl Schwarzschild Strasse 2, 85748 Garching bei München, Germany e-mail: [arenzini; lpasquin]@eso.org

${ }^{7}$ Universidade Federal do Rio Grande do Sul, Departamento de Astronomia, CP 15051, Porto Alegre 91501-970, Brazil e-mail: bica@if.ufrgs.br

8 UCLA, Department of Physics \& Astronomy, 8979 Math-Sciences Building, Los Angeles, CA 90095-1562, USA e-mail: rmr@astro.ucla.edu

Received 10 July 2004 / Accepted 10 January 2005

\begin{abstract}
High resolution spectra of 5 giants, including a horizontal branch star, of the metal-rich globular cluster 47 Tucanae were obtained with the UVES spectrograph at the $8 \mathrm{~m}$ VLT UT2-Kueyen telescope. The atmospheric parameters $\left(T_{\text {eff }}, \log g\right.$, $[\mathrm{Fe} / \mathrm{H}], v_{\mathrm{t}}$ ) were derived from $V I J K$ photometry and spectroscopic data based on Fe I and Fe II lines. Fe I and Fe II iron abundances and respective total errors $[\mathrm{Fe} \mathrm{I} / \mathrm{H}]=-0.66 \pm 0.12$ and $[\mathrm{Fe} \mathrm{II} / \mathrm{H}]=-0.69 \pm 0.24$ are found. Abundances of $\alpha(\mathrm{O}, \mathrm{Mg}, \mathrm{Ca}$, $\mathrm{Si}, \mathrm{Ti})$, odd- $\mathrm{Z}(\mathrm{Na}, \mathrm{Al}), \mathrm{s}-(\mathrm{Ba}, \mathrm{La}, \mathrm{Zr})$, and $r$-process $(\mathrm{Eu})$ elements were determined by means of spectrum synthesis. The main results are $[\mathrm{O} / \mathrm{Fe}]=+0.35 \pm 0.11,[\mathrm{Mg} / \mathrm{Fe}] \approx[\mathrm{Si} / \mathrm{Fe}] \approx[\mathrm{Ti} / \mathrm{Fe}]=+0.23 \pm 0.17,[\mathrm{Ca} / \mathrm{Fe}]=0.0 \pm 0.15,[\mathrm{Ba} / \mathrm{Fe}]=+0.31 \pm 0.22$ and $[\mathrm{Eu} / \mathrm{Fe}]=+0.33 \pm 0.10$. Overabundances of the $\alpha$-elements $\mathrm{O}, \mathrm{Mg}, \mathrm{Si}$ and $\mathrm{Ti}$, and of $\mathrm{Eu}$ are similar to those seen in halo metal-poor stars, whereas a solar Ca-to-Fe ratio resembles the values found in bulge stars. An overall metallicity $Z=0.006$ or $[\mathrm{M} / \mathrm{H}]=-0.45$ is thus obtained, as is a mean heliocentric radial velocity $v_{\mathrm{r}}^{\text {hel }}=-22.43 \pm 1.99 \mathrm{~km} \mathrm{~s}^{-1}$.
\end{abstract}

Key words. globular clusters: individual: 47 Tucanae - globular clusters: general - stars: abundances

\section{Introduction}

47 Tucanae is the second brightest globular cluster of our Galaxy. Its distance is $4.5 \mathrm{kpc}$ (Harris 1996, as updated at http://physun.physics.mcmaster.ca/Globular.html). It is located at a high Galactic latitude J2000 $\alpha=00^{\mathrm{h}} 24^{\mathrm{m}} 05.19^{\mathrm{s}}, \delta=-72^{\circ} 04^{\prime} 49.9^{\prime \prime}\left(l=305.90^{\circ}\right.$, $\left.b=-44.89^{\circ}\right)$, and has a low reddening of $E(B-V)=0.04$ (Harris 1996). As a template metal-rich halo cluster, it is often considered as the prototype of a metal-rich globular cluster. Its metallicity of $[\mathrm{Fe} / \mathrm{H}]=-0.7$ (Harris 1996) is lower than

^ Observations collected both at the European Southern Observatory, Paranal and La Silla, Chile (ESO programmes 65.L-0340, 65.L-0371, 67.D-0489 and 69.D-0582).

$\star \star$ Table A.1 is only available in electronic form at the CDS via anonymous ftp to cdsarc.u-strasbg.fr $(130.79 .128 .5)$ or via http:/kdsweb.u-strasbg.fr/cgi-bin/qcat?J/A+A/435/657 more metal-rich bulge clusters such as NGC 6528, NGC 6553, NGC 6440, Terzan 5 and Liller 1, which show metallicities close to the solar value (e.g. Barbuy et al. 1998, 1999a; Cohen et al. 1999; Carretta et al. 2001; Origlia et al. 2002; Meléndez et al. 2003; Zoccali et al. 2004). These more metal-rich clusters are templates for studies of elliptical galaxies (Bica 1988), whereas 47 Tuc is representative of the metal-rich component of the bimodal metallicity distribution of globular clusters in external galaxies (see Geisler et al. 2002). Despite these interesting characteristics, there are not enough detailed analyses based on high resolution and high S/N CCD spectroscopy of individual member stars available in the literature. The main aim of this work is to derive precise abundances for giants of 47 Tuc, as a reference for other work on metal-rich clusters. To this end, a detailed abundance analysis of five stars in 47 Tuc using high resolution échelle spectra obtained with UVES at the ESO VLT-UT2 Kueyen telescope is presented. 
Brown \& Wallerstein $(1990,1992)$ have used CCD high resolution échelle spectra to analyse 4 stars, two of them located on the Red Giant Branch (RGB) and the other two on the Asymptotic Giant Branch (AGB). After finding a spread in abundances, these authors discuss the possibility of primordial abundance variations. Norris \& Da Costa (1995, hereafter NC95) analysed one cool giant based on high resolution spectra $(R \sim 38000)$, as a comparison object in a study of $\omega$ Centauri giants. Carretta et al. (2004, hereafter C04) used UVES@VLT to derive abundances of turn-off stars, with 3 dwarfs and 9 subgiants; a metallicity $[\mathrm{Fe} / \mathrm{H}]=-0.67$, together with an overabundance of $\alpha$-elements of +0.3 dex were obtained, and no significant differences were found between the pattern shown by giants and their less evolved stars. James et al. (2004, hereafter J04) derived the heavy element abundances using the same spectra studied by $\mathrm{C} 04$.

The observations are described in Sect. 2, while the stellar parameters and abundances are derived in Sect. 3. Abundance ratios are discussed in Sect. 4, and conclusions drawn in Sect. 5.

\section{The data}

\subsection{Imaging}

$B, V$ and $I$ images of 47 Tuc were obtained in June 2002 with the Wide-Field Imager (WFI) at the $2.2 \mathrm{~m}$ ESO-MPI telescope (La Silla, Chile). The data were obtained using our program dedicated to surveying the Galactic globular clusters with the WFI. The WFI camera consists of eight $2048 \times 4096$ EEVCCDs with a total field of view of $34 \times 33 \mathrm{arcmin}^{2}$. The exposure times were divided into deep and shallow in order to sample both bright red giants and the faint main sequence. All scientific images were dithered in such a way as to cover the gaps separating the eight $2048 \times 4096$ CCDs. The seeing conditions were generally good throughout the run. Basic reductions of the CCD mosaic (de-biassing and flat-fielding) were performed using the IRAF package MSCRED (Valdes 1998).

Stellar photometry was performed using the DAOPHOT and ALLFRAME programs (Stetson 1994). Further details are given in Momany et al. (2002). The instrumental PSF magnitudes were normalized to $1 \mathrm{~s}$. exposure and zero airmass. These were then converted into aperture magnitudes assuming that $m_{\text {ap }}=m_{\mathrm{PSF}}-$ const., where the constant is the aperture correction.

The photometric calibrations were defined using standard UBVI stars from Landolt (1992). Secondary standard stars from Stetson (http://cadcwww.hia.nrc.ca/standards/) provided a larger number of standards. In a few cases these belonged to the same globular clusters that were to be calibrated. Calibration uncertainties in the $B V I$ filters are estimated as $0.03,0.03$ and 0.04 , respectively. In deriving the calibration equations we assumed the following extinction coefficients for La Silla: $K_{B}=0.23, K_{V}=0.16$ and $K_{I}=0.07$ (http://www.ls.eso.org/lasilla/atm_ext/). $J, H$ and $K_{\mathrm{S}}$ colours are from the 2MASS Atlas (Skrutskie et al. 1997), http://ipac.caltech.edu/2mass/releases/allsky/. Five individual stars were selected from our sample for spectroscopic observations. The location of target stars on the

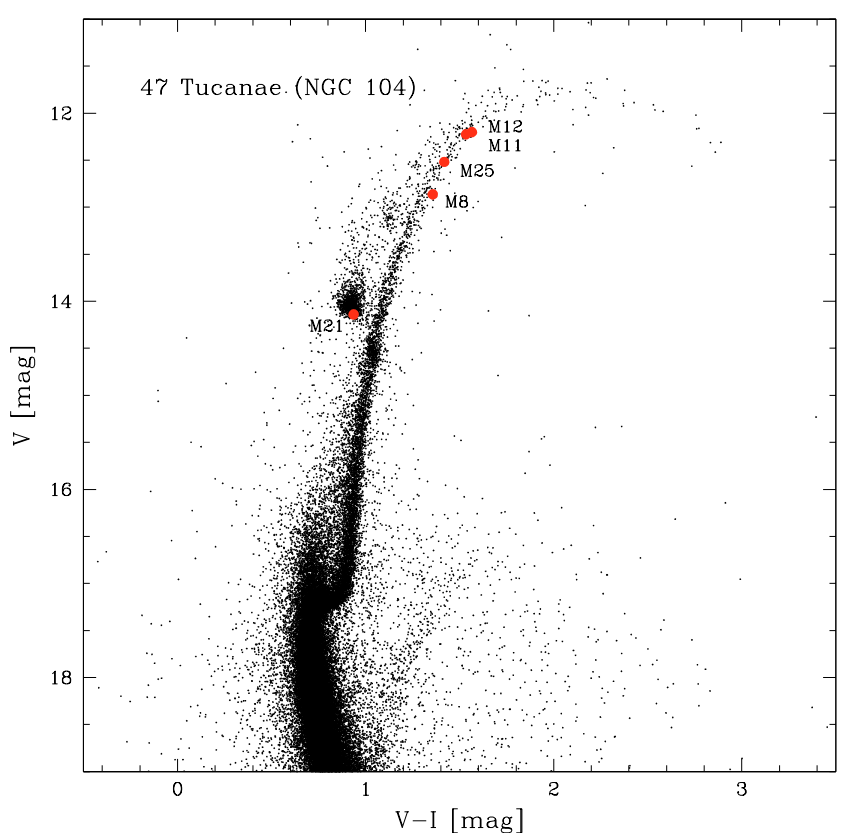

Fig. 1. $V$ vs. $V-I$ Colour-Magnitude Diagram of 47 Tucanae with the target stars indicated.

Table 1. $B V R I$ from ESO-WFI and $J H K_{\mathrm{S}}$ from 2MASS. $V$ and $I$ are in the Cousins system.

\begin{tabular}{lcccccc}
\hline \hline & $B$ & $V$ & $I$ & $J$ & $H$ & $K \mathrm{~s}$ \\
\hline M 8 & 13.839 & 12.518 & 11.100 & 10.028 & 9.247 & 9.094 \\
M 11 & 13.636 & 12.201 & 10.636 & 9.551 & 8.666 & 8.533 \\
M 12 & 13.885 & 12.224 & 10.690 & 9.612 & 8.901 & 8.639 \\
M 21 & 14.969 & 14.140 & 13.204 & 12.479 & 11.993 & 11.953 \\
M 25 & 14.115 & 12.897 & 11.559 & 10.544 & 9.865 & 9.709 \\
\hline
\end{tabular}

Colour-Diagram Magnitude (CMD) is shown in Fig. 1, while Table 1 lists the magnitudes of the sample stars. The star's identifications follow the notation of Menzies (1973).

\subsection{Spectra}

High resolution spectra for five giants of 47 Tuc in the wavelength range $\lambda \lambda$ 4800-6800 $\AA$ were obtained with the UVES spectrograph at the ESO VLT. The red portion of the spectrum (5800-6800 ̊) was obtained with the MIT backside illuminated ESO CCD \# 20, of $4096 \times 2048$ pixels, and pixel size $15 \times 15 \mu \mathrm{m}$. The blue portion of the spectrum (4800$5800 \AA$ A) uses ESO Marlene EEV CCD-44, backside illuminated, $4102 \times 2048$ pixels, and pixel size $15 \times 15 \mu \mathrm{m}$. With the UVES standard setup 580, resolution is $R \sim 45000$ for a 1 -arcsec slit width, while $R \sim 55000$ for a slit of 0.8 arcsec. The pixel scale is $0.0147 \AA / \mathrm{px}$. Table 2 shows the log of observations.

The spectra were reduced using the UVES context of the MIDAS reduction package, including bias and inter-order background subtraction, flatfield correction, extraction and wavelength calibration (Ballester et al. 2000). Figure 2 illustrates the quality of the spectra for the 5 sample stars. 
Table 2. Log of the spectroscopic observations carried out in 24-26 June 2000 and 6 July 2001. The quoted seeing are mean values along the exposures. For star M21 the $S / N$ was obtained based on a spectrum smoothed with a gaussian of $\sigma=3$ pixels.

\begin{tabular}{|c|c|c|c|c|c|c|c|c|c|c|c|}
\hline star & $\alpha_{2000}$ & $\delta_{2000}$ & $\begin{array}{c}\text { date/ } \\
\text { Julian Day }\end{array}$ & UT & $\begin{array}{l}\exp \\
(\min )\end{array}$ & $\begin{array}{l}\text { Seeing } \\
\left({ }^{\prime \prime}\right)\end{array}$ & Airmass & $(S / N) / \mathrm{px}$ & $\begin{array}{c}\text { Slit } \\
\text { width }\end{array}$ & $\begin{array}{c}v_{\mathrm{r}}^{\mathrm{obs}} \\
\mathrm{km} \mathrm{s}^{-1}\end{array}$ & $\begin{array}{c}v_{\mathrm{r}}^{\text {hel. }} \\
\mathrm{km} \mathrm{s}^{-1}\end{array}$ \\
\hline \multirow[t]{2}{*}{ M 8} & $00: 26: 28.8$ & $-71: 50: 12$ & $\begin{array}{l}24.06 .00 \\
2451719\end{array}$ & 09:31 & 45 & 1.2 & 1.5 & 280@6218Å & 0.8 & -34.67 & -28.16 \\
\hline & & & $\begin{array}{l}04.07 .01 \\
2452094\end{array}$ & 09:24 & 30 & 0.8 & “ & 245@6461§ & 0.8 & -34.67 & -28.16 \\
\hline M 11 & $00: 26: 57.7$ & $-71: 49: 10$ & $\begin{array}{l}04.07 .01 \\
2452094\end{array}$ & $09: 43$ & 25 & 0.8 & “ & $241 @ 6329 \AA$ & 0.8 & -25.89 & -19.29 \\
\hline M 12 & $00: 26: 27.1$ & $-71: 47: 40$ & $\begin{array}{l}04.07 .01 \\
2452094\end{array}$ & 09:04 & 15 & 0.8 & “ & 247@6329尺 & 0.8 & -29.34 & -22.80 \\
\hline M 21 & $00: 25: 57.2$ & $-71: 49: 28$ & $\begin{array}{l}05.07 .01 \\
2452095\end{array}$ & 09:22 & 35 & 1.5 & “ & 213@6460Å & 0.8 & -23.28 & -16.97 \\
\hline M 25 & $00: 24: 47.2$ & $-71: 47: 44$ & $\begin{array}{l}25.06 .00 \\
2451720\end{array}$ & 09:46 & 30 & 1.5 & “ & 258@6401Å & 1.0 & -33.19 & -24.96 \\
\hline
\end{tabular}

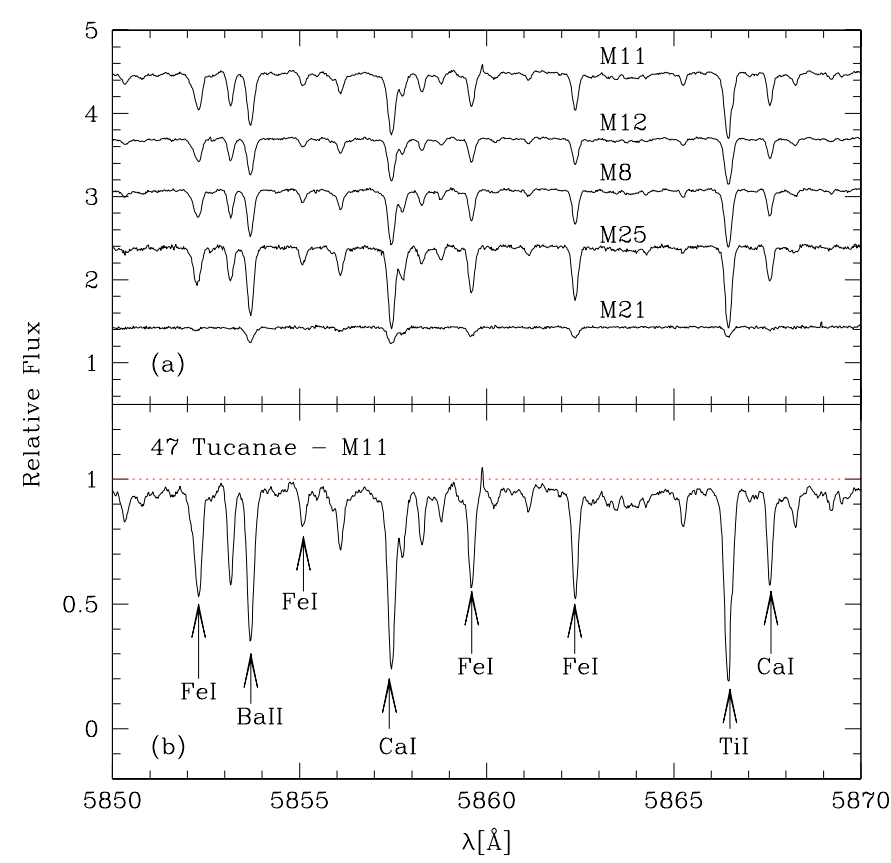

Fig. 2. a) Typical spectra of the 47 Tucanae giants analysed in this work; and b) some atomic lines identified in a 20 Å region of 47 Tuc: M 11.

Radial velocities were derived using the IRAF tasks rvidlines and rvcorrect, based on the use of 40 reference lines. A mean $v_{\mathrm{r}}=-29.27 \pm 0.07 \mathrm{~km} \mathrm{~s}^{-1}$ or heliocentric $v_{\mathrm{r}}^{\text {hel }}=$ $-22.43 \pm 0.07 \mathrm{~km} \mathrm{~s}^{-1}$ ( $\mathrm{rms} \sigma=4.44 \mathrm{~km} \mathrm{~s}^{-1}$, and mean standard deviation $\sigma / \sqrt{N}=1.99 \mathrm{~km} \mathrm{~s}^{-1}$ ) was found for 47 Tuc, which is in good agreement with the values of $17 \pm 1.5 \mathrm{~km} \mathrm{~s}^{-1}$ (rms $\sigma=10 \mathrm{~km} \mathrm{~s}^{-1}$ ) from a measurement of 47 stars by Minniti (1995a) and $-18.7 \pm 0.2 \mathrm{~km} \mathrm{~s}^{-1}$ given by Harris (1996) and Rutledge et al. (1997).

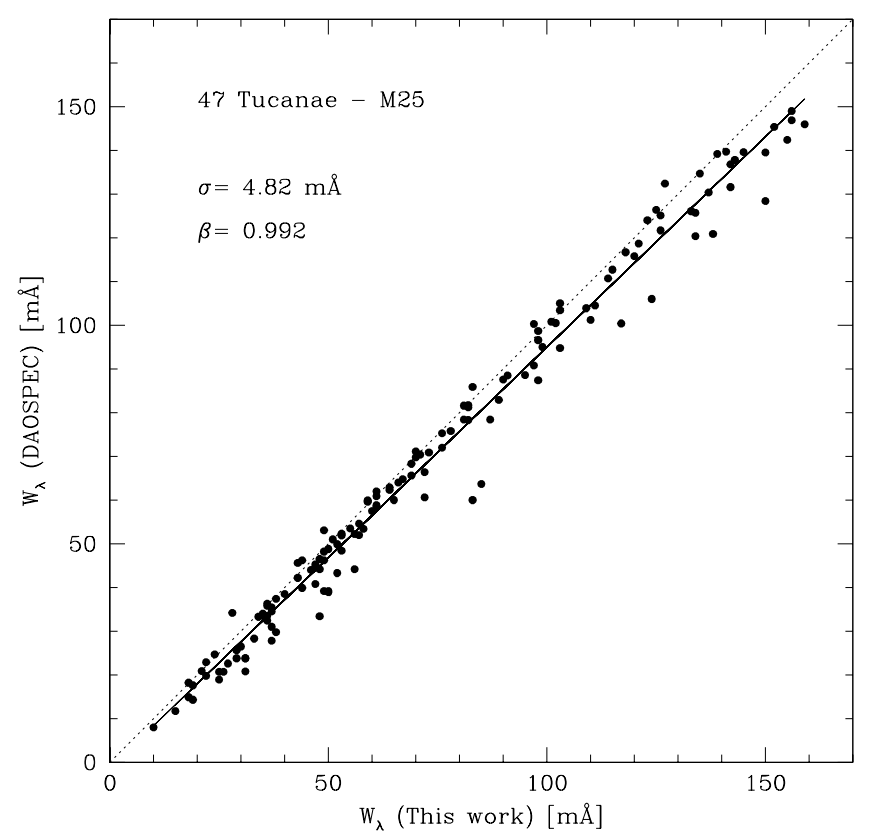

Fig. 3. Comparison between equivalent widths $W_{\lambda}(\mathrm{m} \AA)$ of Fe I lines for star M 25, obtained with the code DAOSPEC (P. Stetson \& E. Pancino 2005, in preparation), and measured line-by-line with IRAF. The solid line is a linear regression to the data, and the dotted line corresponds to the case where the two sets of $W_{\lambda}$ 's are identical. Standard deviation $(\sigma)$ of the regression and correlation coefficient $(\beta)$ of the fit are given.

\section{Stellar parameters}

\subsection{Equivalent widths and oscillator strengths}

The equivalent widths $W_{\lambda}$ were measured using IRAF fitting a Gaussian profile to weak lines, a multi-Gaussian fit to blends, and Voigt profiles to strong lines. We also measured equivalent widths using a new automatic code developed by P. Stetson \& E. Pancino (DAOSPEC, Stetson \& Pancino 2005, in prep., 
Table 3. Dereddened colours. J: Johnson, TCS: Telescope Carlos Sanches, C: Cousins, CIT: California Institute Technology.

\begin{tabular}{lcccccc}
\hline \hline Star & $\begin{array}{c}(V-I)_{0} \\
(\mathrm{~J})\end{array}$ & $\begin{array}{c}(V-K)_{0} \\
(\mathrm{TCS})\end{array}$ & $(J-K)_{0}$ & $(V-I)_{0}$ & $(V-K)_{0}$ & $(J-K)_{0}$ \\
& & & $(\mathrm{C})$ & $(\mathrm{CIT})$ & $(\mathrm{CIT})$ \\
\hline M 8 & 1.754 & 3.306 & 0.873 & 1.365 & 3.290 & 0.875 \\
M 11 & 1.943 & 3.550 & 0.954 & 1.512 & 3.534 & 0.955 \\
M 12 & 1.903 & 3.467 & 0.911 & 1.481 & 3.451 & 0.912 \\
M 21 & 1.134 & 2.072 & 0.481 & 0.883 & 2.053 & 0.489 \\
M 25 & 1.654 & 3.071 & 0.778 & 1.284 & 3.054 & 0.782 \\
\hline
\end{tabular}

http://cadcwww.hia.nrc.ca/stetson/daospec/), shown in Fig. 3 for $10<W_{\lambda}<160 \mathrm{~m} \AA$.

The Fe I line list and respective oscillator strengths from the National Institute of Standards \& Technology (NIST) library (Martin et al. 2002) were adopted, along with Fe II oscillator strengths renormalized by Meléndez \& Barbuy (2005). The list of Fe I and Fe II lines is given in Table A.1.

Damping constants for neutral lines were computed using the collisional broadening theory of Barklem et al. (1998, 2000, and references therein), as described in Zoccali et al. (2004) and Coelho et al. (2005). The oscillator strengths for elements other than $\mathrm{Fe}$ were obtained by fitting the solar spectrum observed with the same VLT-UVES instrumentation (www.eso.org/observing/dfo/quality/UVES/pipeline/ solar_spectrum.html) and the Arcturus spectrum (Hinkle et al. 2000) for different literature $g f$-values, as described in Zoccali et al. (2004). The adopted $g f$-values were selected by choosing the best fit to the solar and Arcturus spectra and are indicated in Table 6. In order to illustrate the reliability of the atomic constants, in Fig. 7 we show the CaI $6455.5 \AA$ line for the solar, Arcturus, and 47 Tuc: M 12 spectra. Table 6 also reports $g f$-values used by Brown \& Wallerstein (1992), in order to assess possible abundance discrepancies resulting from differences in $g f$-values. For the oxygen forbidden line [OI] $6300.311 \AA$ we adopt the oscillator strength derived by Allende Prieto et al. (2001) of log $g f=-9.716$. The NiI line that blends the $[\mathrm{OI}]$ line is included in the line list, but its effect is negligible in $\mathrm{K}$ giants.

For lines of the heavy elements BaII, LaII, and EuII, a hyperfine structure was taken into account, based on the hyperfine constants by Lawler et al. (2001a) for EuII, Lawler et al. (2001b) for LaII, and Biehl (1976) for BaII, where a solar isotopic mix is adopted, and a code described and made available by McWilliam (1998) is employed.

Solar abundances were adopted from Grevesse \& Sauval (1998), except that of oxygen, where the value $\epsilon(\mathrm{O})=8.77$ of Allende Prieto et al. (2001), suitable for the use of 1-D model atmospheres was used. For europium, the updated value is $\epsilon(\mathrm{Eu})=0.54$.

\subsection{Temperatures}

A reddening $E(B-V)=0.04$ for 47 Tuc (Harris 1996) and reddening laws $E(V-I) / E(B-V)=1.33$ (Dean et al. 1978), $E(V-K) / E(B-V)=2.744$, and $E(J-K) / E(B-V)=0.52$
(Rieke \& Lebofsky 1985) were all adopted, and the dereddened colours are given in Table 3.

Photometric temperatures were derived from $V, I, J, K$ magnitudes, adopting the colour-temperature calibrations of Alonso et al. (1999, 2001 hereafter AAM99) and Houdashelt et al. (2000, hereafter HBS00). Table 4 gives the effective temperatures derived from $V-I, V-K$, and $J-K$ colours using the two calibrations. The photometric $T_{\text {eff }}$ values given by the $V-K$ colour using the AAM99 calibration were adopted, as representative of the photometric indicators. The effective temperatures were then checked by imposing excitation equilibrium for $\mathrm{Fe} \mathrm{I}$ and $\mathrm{Fe}$ II lines of different excitation potential, as illustrated in Fig. 4 and given in Table 5. Spectroscopic temperatures are about $50 \mathrm{~K}$ hotter than photometric ones. For the hotter Horizontal Branch (HB) star M 21, we were able to derive the temperature from the $\mathrm{H} \alpha$ profile $T(\mathrm{H} \alpha)$ (Fig. 5).

\subsection{Gravities}

The classical relation $\log g_{*}=4.44+4 \log \left(T_{*} / T_{\odot}\right)+0.4\left(M_{\mathrm{bol}}-\right.$ $4.75)+\log \left(M_{*} / M_{\odot}\right)$ was used, adopting $T_{\odot}=5780 \mathrm{~K}, M_{*}=$ $0.80 M_{\odot}$ and $M_{\text {bol } \odot}=4.75$ (Cram 1999). A distance modulus of $(m-M)_{V}=13.27$ (Zoccali et al. 2001), a reddening of $E(B-V)=0.04$ and $A_{\mathrm{V}}=0.124$, together with bolometric correction $\mathrm{BC}_{V}$ values from AAM99 were used to derive photometric gravities, from the classical formula above, which are given in Tables 4 and 5. We also derived $\log g$ from $12.5 \mathrm{Gyr}$ isochrones of $Z=0.0054, Y=0.24,[\mathrm{Fe} / \mathrm{H}]=-0.76$, and $[\alpha / \mathrm{Fe}]=+0.3$ by Kim et al. (2002). These gravity values, reported in Table 4, are higher than the photometric ones derived by using the classical relation, by $\Delta \log g=0.11$. Finally, we derived $\log g$ from ionization equilibrium of $\mathrm{Fe} \mathrm{I}$ and $\mathrm{Fe}$ II lines (Table 4). In the following, the spectroscopic effective temperatures and gravities are assumed.

\subsection{Iron abundance}

The set of equivalent widths measured with IRAF were used in the following, and Fe I and Fe II lines with $10<W_{\lambda}<$ $160 \mathrm{~m} \AA$ were selected. Photospheric 1D models for the sample giants were extracted from the NMARCS grid (Plez et al. 1992) originally developed by Bell et al. (1976) and Gustafsson et al. (1975). The LTE abundance analysis and spectrum synthesis calculations were performed with the codes by Spite (1967, and subsequent improvements in the last thirty years), described in Cayrel et al. (1991) and Barbuy et al. (2003).

Stellar parameters were derived by initially adopting the photometric effective temperature and gravity, and then further constraining both the temperature by imposing excitation equilibrium for Fe I lines and the gravity by imposing ionization equilibrium for Fe I and Fe II. Microturbulence velocity $v_{\mathrm{t}}$ was determined by canceling the trend of $\mathrm{Fe} I$ abundance vs. equivalent width. Figures $6 \mathrm{a}, \mathrm{b}$ show the derived $\mathrm{Fe}$ abundances as a function of reduced equivalent width for stars M 8 and M 11.

The final values used in the analysis are the spectroscopic parameters $T_{\text {eff }}, \log g, v_{\mathrm{t}},[\mathrm{Fe} \mathrm{I} / \mathrm{H}]$ and $[\mathrm{Fe} \mathrm{II} / \mathrm{H}]$ values reported in Table 5, with the exception of $T_{\text {eff }}=T(\mathrm{H} \alpha)$ adopted for 
Table 4. Photometric effective temperatures $T_{\text {eff }}$ derived using $V-I, V-K$ and $J-K$ based on relations by AAM99 and HSB00, absolute magnitudes $M_{V}$, bolometric corrections $B C_{V}$, bolometric magnitudes $M_{\text {bol }}$ and gravity values derived.

\begin{tabular}{|c|c|c|c|c|c|c|c|c|c|c|c|c|c|c|}
\hline \multirow[b]{2}{*}{ Star } & \multicolumn{4}{|c|}{ AAM99 } & \multicolumn{4}{|c|}{ HBS00 } & \multicolumn{4}{|c|}{ AAM99 } & \multirow[b]{2}{*}{$\log g_{\mathrm{Kim}}$} & \multirow[b]{2}{*}{$\log g_{\text {ion }}$} \\
\hline & $T_{V-I}$ & $T_{V-K}$ & $T_{J-K}$ & $\langle T\rangle$ & $T_{V-I}$ & $T_{V-K}$ & $T_{J-K}$ & $\langle T\rangle$ & $M_{V}$ & $\mathrm{CB}(\mathrm{V})$ & $M_{\mathrm{bol}}$ & $\log g_{\text {photo }}$ & & \\
\hline M 8 & 4106 & 4036 & 3970 & $4037 \pm 39$ & 4150 & 4082 & 3956 & $4063 \pm 57$ & -0.700 & -0.869 & -1.569 & 1.19 & 1.29 & 1.48 \\
\hline M11 & 3933 & 3920 & 3813 & $3888 \pm 38$ & 4003 & 3972 & 3767 & $3914 \pm 74$ & -1.017 & -1.025 & -2.042 & 0.95 & 1.03 & 1.20 \\
\hline M12 & 3966 & 3957 & 3895 & $3939 \pm 22$ & 4028 & 4006 & 3866 & $3966 \pm 51$ & -0.994 & -0.971 & -1.965 & 1.00 & 1.02 & 1.45 \\
\hline M21 & 5000 & 5017 & 5099 & $5038 \pm 30$ & 5080 & 5091 & 5163 & $5111 \pm 26$ & +0.922 & -0.263 & +0.659 & 2.46 & - & 2.46 \\
\hline M25 & 4217 & 4166 & 4181 & $4188 \pm 15$ & 4259 & 4216 & 4201 & $4225 \pm 17$ & -0.321 & -0.734 & -1.055 & 1.45 & 1.57 & 1.65 \\
\hline
\end{tabular}
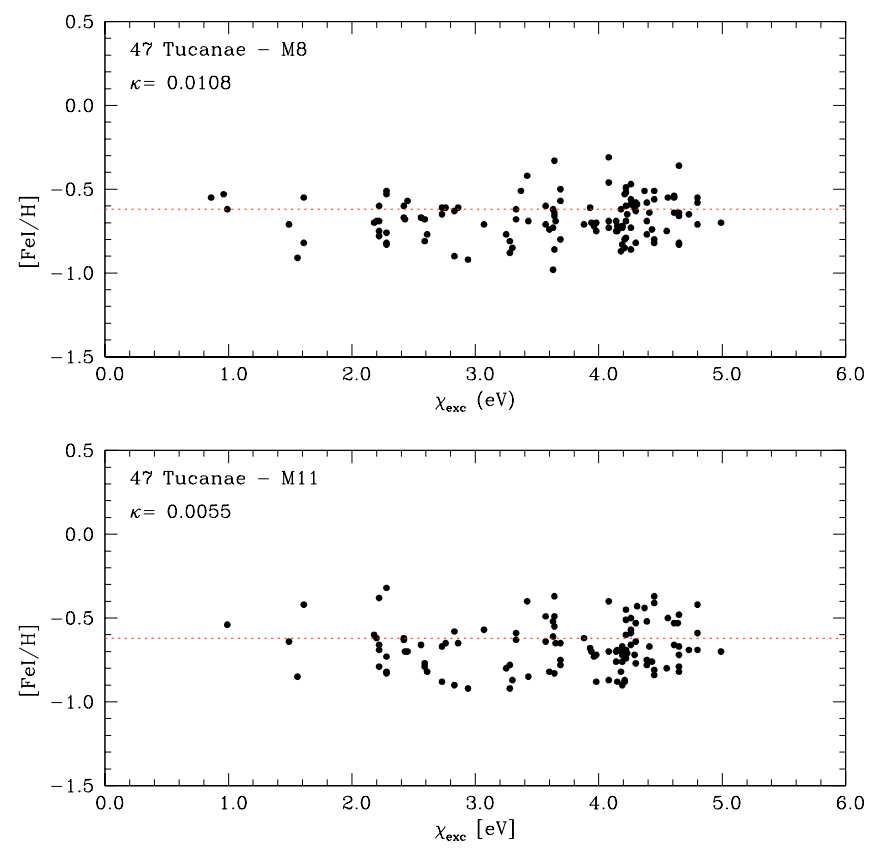

Fig. 4. Excitation equilibrium temperature derived by imposing a constant abundance of Fe I as a function of the excitation potential $\chi_{\mathrm{ex}}(\mathrm{eV})$, for stars M 8 and M 11.

$\mathrm{M} 21$. The mean iron abundances derived are $[\mathrm{Fe} \mathrm{I} / \mathrm{H}]=-0.66$ and $[\mathrm{Fe} \mathrm{II} / \mathrm{H}]=-0.69$, with the latter value adopted in the following Sections.

\subsection{Abundance ratios}

Elemental abundances were obtained through line-by-line spectrum synthesis calculations for the line list given in Table 6 . The calculations of synthetic spectra were carried out using the code described in Barbuy et al. (2003), where constants for atomic lines are outlined in Sect. 3.1 and molecular lines of $\mathrm{CN} \mathrm{A}^{2} \Pi-\mathrm{X}^{2} \Sigma, \mathrm{C}_{2}$ Swan $\mathrm{A}^{3} \Pi-\mathrm{X}^{3} \Pi$, TiO $\mathrm{A}^{3} \Phi-\mathrm{X}^{3} \Delta \gamma$ and $\mathrm{B}^{3} \Pi-\mathrm{X}^{3} \Delta \gamma^{\prime}$ systems are taken into account.

Table 7 gives the final mean results for each star and the mean for the 5 stars, whereas Table 8 reports the errors (Sect. 3.6). The results given in Table 7 indicate that the $\alpha$-elements $\mathrm{Mg}, \mathrm{Si}$, and $\mathrm{Ti}$ are enhanced by $[\mathrm{Mg}, \mathrm{Si}$, $\mathrm{Ti} / \mathrm{Fe}] \approx+0.25$, whereas $[\mathrm{O} / \mathrm{Fe}]=+0.35$, and $[\mathrm{Ca} / \mathrm{Fe}] \approx 0.0$. Figure $8 \mathrm{~b}$ illustrates the fit of synthetic spectra to the observed one, whereas Fig. 8a shows the location of telluric lines, indicating that the $[\mathrm{OI}] 6300 \AA$ line is free of blending with them. The r-process element Europium is overabundant, with $[\mathrm{Eu} / \mathrm{Fe}]=0.33$, compatible with the high $\alpha$-element enhancements.

The abundance of $\mathrm{Na}$ derived from the NaI 5688.205 line is not considered in the final mean abundance, given that it is clearly subject to non-LTE effects (Gratton et al. 1999), whereas such effects are negligible on the NaI 6154/6161 doublet (Takeda et al. 2003). The Ba abundance for M21 was not considered in the final mean for the cluster of $[\mathrm{Ba} / \mathrm{Fe}]=0.31$, since it appears anomalous. [La, $\mathrm{Zr} / \mathrm{Fe}] \approx 0.0$, as expected for s-elements in old populations.

Table 9 compares the present results and those of BW92, NC95, C04 and J04. There is good agreement with C04, as well as with J04 for Ba and Eu, particularly Ba. Note that the results by $\mathrm{J} 04$ were reported separately for subgiants and turn-off stars, and we adopted a mean of their results. The differences seen with BW92 appear to be due mostly to their lower iron abundance, but also to differences in adopted $g f$-values (see Table 6). NC95 indicated enhancements of $\alpha$-elements, in rather good agreement with the present results. However their Eu abundance clearly disagrees with BW92, J04, and our results. NC95 also find very low $[\mathrm{Eu} / \mathrm{Fe}]$ values for their $\omega$ Cen giants; therefore, there is probably a mistaken oscillator strength used in their calculations.

\subsection{Errors}

\subsubsection{Equivalent widths}

Figure 3 shows the dispersion $\sigma=4.82 \mathrm{~m} \AA$ between the sets of equivalent widths measured with DAOSPEC and IRAF. After comparing the set of equivalent widths for two sample stars with similar atmospheric parameters $\left(T_{\mathrm{eff}}: \log g:[\mathrm{Fe} / \mathrm{H}]\right), \mathrm{M} 8$ (4086:1.48:-0.62) and M 12 (4047:1.45:-0.63), $\sigma=4.55 \mathrm{m \AA}$ is obtained. Supposing that the two sets of measurements are susceptible to the same error sources, a typical uncertainty in the equivalent widths of $\sigma=3.22 \mathrm{~m} \AA$ is deduced, which can be taken as a realistic estimation of uncertainty for all stars in the sample.

Using the Cayrel (1988) equation, $\sigma_{W_{\lambda}}=\frac{1.5}{S / N} \sqrt{\omega \delta x}$, (where $\sigma_{W_{\lambda}}$ is the uncertainty for $W_{\lambda}, S / N$ [pix $\left.{ }^{-1}\right]$ the signalto-noise ratio, $\omega[\AA]$ the $F W H M$, and $\delta x$ the pixel size), for typical values of this work, we estimate an uncertainty of $\sigma_{W_{\lambda}}=0.3 \mathrm{m \AA}$. This represents an uncertainty smaller than $3 \%$ 
Table 5. Final atmospheric parameters.

\begin{tabular}{|c|c|c|c|c|c|c|c|c|c|c|}
\hline \multirow[b]{2}{*}{ Star } & \multicolumn{4}{|c|}{ Photometric } & \multicolumn{6}{|c|}{ Spectroscopic } \\
\hline & $\begin{array}{c}T \\
(\mathrm{~K})\end{array}$ & $\begin{array}{c}\log g \\
\left(\mathrm{~cm} \mathrm{~s}^{-2}\right)\end{array}$ & $\begin{array}{c}{[\mathrm{Fe} / \mathrm{H}]} \\
(\mathrm{I})\end{array}$ & $\begin{array}{c}\mathrm{Fe} / \mathrm{H}] \\
(\mathrm{II})\end{array}$ & $\begin{array}{c}v_{\mathrm{t}} \\
\left(\mathrm{km} \mathrm{s}^{-1}\right)\end{array}$ & $\begin{array}{l}T_{\mathrm{exc}} \\
(\mathrm{K})\end{array}$ & $\begin{array}{l}T_{\mathrm{H}_{\alpha}} \\
(\mathrm{K})\end{array}$ & $\begin{array}{c}\log g \\
\left(\mathrm{~cm} \mathrm{~s}^{-2}\right)\end{array}$ & $\begin{array}{c}{[\mathrm{Fe} / \mathrm{H}]} \\
(\mathrm{I})\end{array}$ & $\begin{array}{c}\mathrm{Fe} / \mathrm{H}] \\
(\mathrm{II})\end{array}$ \\
\hline M 8 & 4036 & 1.19 & -0.67 & -0.75 & 1.42 & 4086 & - & 1.48 & -0.62 & -0.65 \\
\hline M 11 & 3920 & 0.95 & -0.67 & -0.72 & 1.49 & 3945 & - & 1.20 & -0.62 & -0.62 \\
\hline M 12 & 3957 & 1.00 & -0.71 & -0.79 & 1.45 & 4047 & - & 1.45 & -0.63 & -0.68 \\
\hline M 21 & 5017 & 2.46 & -0.79 & -0.81 & 1.42 & 5032 & 5100 & 2.46 & -0.77 & -0.82 \\
\hline M 25 & 4166 & 1.45 & -0.67 & -0.73 & 1.37 & 4200 & - & 1.65 & -0.64 & -0.67 \\
\hline
\end{tabular}

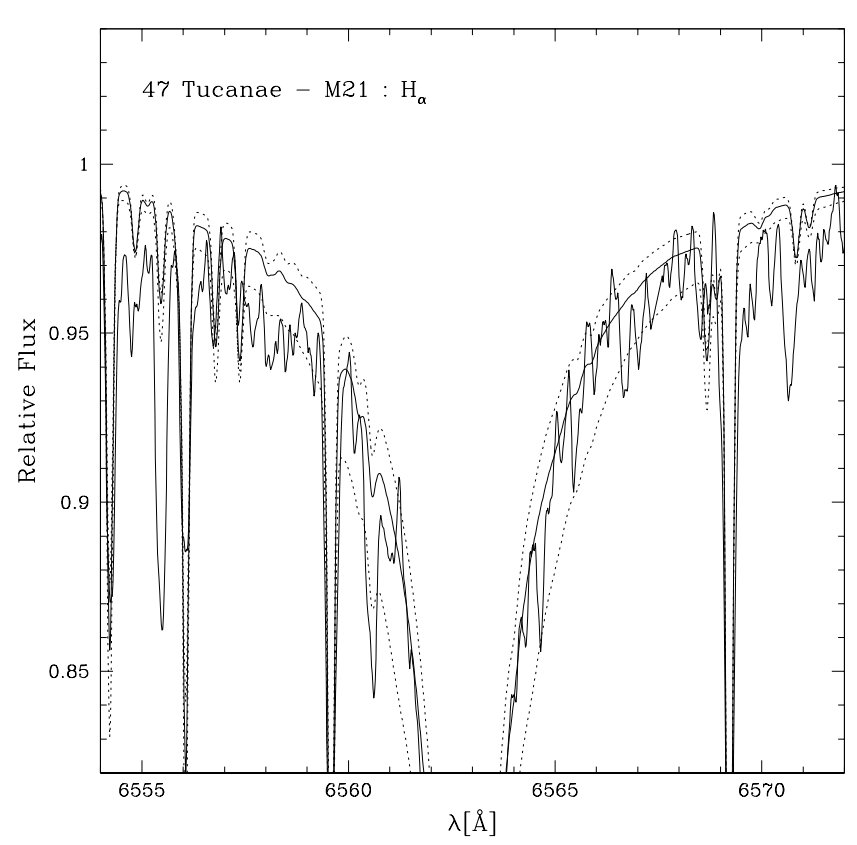

Fig. 5. $\mathrm{H} \alpha$ profile for the star M 21 showing the wings for $T_{\text {eff }}=5000$, 5100 (best fit), and $5200 \mathrm{~K}$.

for the weaker lines $\left(W_{\lambda}=10 \mathrm{~m} \AA\right)$ and smaller than $1 \%$ for the stronger lines $\left(W_{\lambda}=160 \mathrm{~m} \AA\right)$. Note, however, that the equation above does not consider uncertainties regarding the precise location of continuum, such that the value of $\sigma_{W_{\lambda}}$ is underestimated.

\subsubsection{Atmospheric parameters}

(i) $T_{\text {eff }}$ : the differences in effective temperatures derived from the different indicators (Tables 4 and 5) show errors within $\pm 100 \mathrm{~K}$. (ii) $\log g$ : the surface gravity $\log g\left(T_{\mathrm{eff}}, M_{*}, M_{\mathrm{bol}}\right)$ is affected by errors on $T_{\text {eff }}\left(\sigma_{T_{\text {eff }}}=100 \mathrm{~K}\right), M_{*}\left(\sigma_{M_{*}}=0.1 M_{\odot}\right)$, and $M_{\text {bol }}\left(\sigma_{M_{\text {bol }}}=0.07 \mathrm{mag}\right)$, giving \pm 0.10 dex. By considering the mean differences between the photometric and spectroscopic gravities (Table 4), the uncertainty on $\log g$ is \pm 0.2 dex. (iii) $v_{\mathrm{t}}$ : the rms of the data points of excitation equilibrium (Fig. 6) shows an uncertainty of the order of $0.2 \mathrm{~km} \mathrm{~s}^{-1}$ on the microturbulence velocity. (iv) $[\mathrm{Fe} / \mathrm{H}]$ : Table 8 reports the uncertainties on the derivation of abundances, obtained by
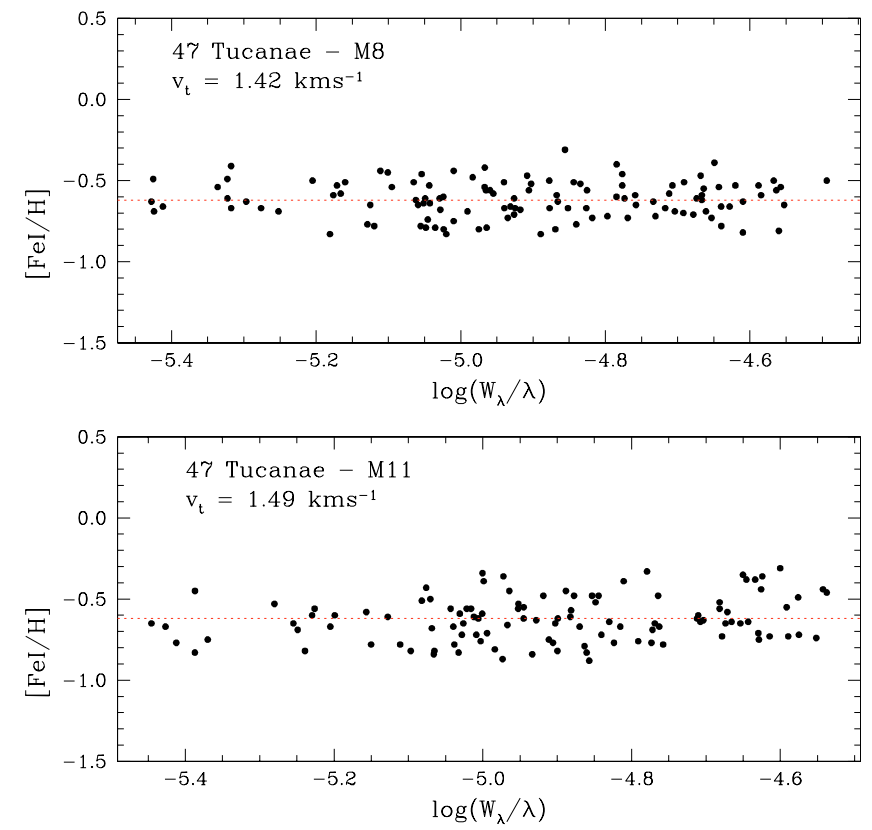

Fig. 6. $[\mathrm{Fe} \mathrm{I/H]} \mathrm{vs.} \mathrm{reduced} \mathrm{equivalent} \mathrm{width} \mathrm{for} \mathrm{stars} \mathrm{M} 8$ and M 11 . The dotted line corresponds to mean abundance obtained.

computing the results for $\Delta T_{\text {eff }}=100 \mathrm{~K}, \Delta \log g=0.2$ and $\Delta v_{\mathrm{t}}=0.2 \mathrm{~km} \mathrm{~s}^{-1}$. The total error is given in the last column.

\section{Discussion}

Zinn (1985) proposed subdividing the globular cluster system into two subsystems: (i) the halo clusters, more metal-poor than $[\mathrm{Fe} / \mathrm{H}]<-0.8$, with a spherical distribution and a small rotational velocity, and (ii) the disk clusters containing those of $[\mathrm{Fe} / \mathrm{H}]>-0.8$ with a highly flattened distribution. In his study, 47 Tuc is classified as a disk cluster. Armandroff (1989) argued that the metal-rich clusters are associated with the thick disk. More recently, Minniti (1995b) and Côté (1999) argued that based on their dynamics the metal-rich clusters in the inner spheroid are associated with the galactic bulge rather than with the disk, as already proposed earlier by Frenk \& White (1982). Barbuy et al. (1999b) discussed the properties of clusters within $4 \mathrm{kpc}$ of the Galactic bulge. In the bulge and halo subdivision, 47 Tuc would rather belong to the halo system. It is located far from the bulge at $R_{\mathrm{GC}}=7.4 \mathrm{kpc}$ and is high in 
Table 6. Species, wavelength, excitation potential, $g$-values from Brown \& Wallerstein (1992), and the $g f$-values adopted here, with line-byline abundance ratios. References in Col. 6 correspond to those from where the $g f$-values, given in Col. 5, were adopted. 1: Allende Prieto et al. (2001); 2: NIST; 3: Barbuy et al. (1999); 4: Bensby et al. (2003); 5: McWilliam \& Rich (1994); 6: Brown \& Wallerstein (1992); 7: Kraft (1998); 8: McWilliam (1998); 9: Rutten (1978); 10: Lawler et al. (2001a,b). *: Abundances from the NaI $5688.205 \AA$ line were not considered in the final mean abundances given in Tables 7 and 9 (see Sect. 3.5).

\begin{tabular}{|c|c|c|c|c|c|c|c|c|c|c|}
\hline \multirow[b]{2}{*}{ Species } & \multirow[b]{2}{*}{$\lambda(\AA)$} & \multirow[b]{2}{*}{$\chi_{\mathrm{ex}}(\mathrm{eV})$} & \multicolumn{2}{|c|}{$\log g f$} & \multirow[b]{2}{*}{ Ref. } & \multicolumn{5}{|c|}{$[\mathrm{X} / \mathrm{Fe}]$} \\
\hline & & & BW92 & This & & 48 & M 11 & M 12 & M 21 & 125 \\
\hline O I & 6300.311 & 0.000 & -9.750 & -9.717 & 1 & +0.36 & +0.22 & +0.50 & +0.31 & +0.35 \\
\hline O I & 6363 & 0.020 & --- & -10.250 & 2 & & +0.22 & +0.50 & --- & +0.35 \\
\hline $\mathrm{Na} \mathrm{I}^{*}$ & 5688.205 & 2.104 & -0.370 & -0.457 & 2 & 0.22 & +0.32 & -0.03 & +0.12 & -0.07 \\
\hline $\mathrm{Na} \mathrm{I}$ & 6154.225 & 2.102 & -1.530 & -1.570 & 5 & & & & - & \\
\hline $\mathrm{Na} \mathrm{I}$ & 6160.747 & 2.104 & -1.230 & -1.270 & 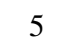 & & & +0.05 & -0.15 & \\
\hline 10 & 6 & 314 & -1.320 & -1.4 & 5 & & & - & - & \\
\hline & 6698.667 & 3.143 & -1.620 & -1.7 & 5 & & +0.13 & +0.03 & - & \\
\hline Ig I & 6319.242 & 5.110 & - & -2.360 & 3 & & & +0.27 & - & \\
\hline $\operatorname{lig} 1$ & 6 & 5.7 & - & -1 & 5 & & & & - & \\
\hline & & & 1.870 & -1 & 2 & & & & & \\
\hline & & 5. & & & 2 & & & +0.35 & & +0.35 \\
\hline & & 5.620 & -1.390 & -1 & 3 & & +0.25 & +0.17 & & +0.17 \\
\hline & & 5.6 & -1.150 & -1 . & 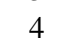 & & . & . & +0.31 & . \\
\hline & & & - & -1 . & 7 & & & +0.10 & +0.31 & +0.2 \\
\hline & & & & & 3 & & & - & - & - \\
\hline & & 1.8 & 0 & & 2 & & & -0.01 & +0.19 & -0.06 \\
\hline & 0 & 25 & - & -2 . & 3 & & & & - & +0.02 \\
\hline & & & 1.030 & -1 . & 2 & & & & 19 & -0.16 \\
\hline & & & - & & - & & & & & \\
\hline & & & & & 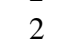 & & & & & -0.16 \\
\hline & 2 & 2523 & -0.800 & & 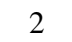 & & +0.04 & & & +0.03 \\
\hline & & & 0 & -0 . & 2 & & & & & \\
\hline & & & & & 4 & & & & & \\
\hline & & & 40 & & 2 & & & & +0.03 & -0.08 \\
\hline & & & - & & 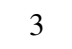 & & & & - & - \\
\hline & & 0 & 0.690 & & 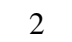 & & -0.11 & -0.06 & +0.14 & -0.21 \\
\hline & & & & & 5 & & & +0.09 & - & \\
\hline & & 00 & 0 & 4 & 2 & & - & 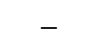 & & +0.21 \\
\hline & & & & & 2 & & & & & +0.53 \\
\hline & & & & & 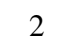 & & +0.28 & +0.33 & & +0.23 \\
\hline & & & 10 & & 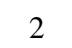 & & & +0.13 & +0.08 & +0.08 \\
\hline & & & & -0 & 2 & & +0.08 & +0.13 & +0.18 & +0.13 \\
\hline $\mathrm{Ti}$ & 6 & 18 & 10 & -1 & 2 & & - & - & - & - \\
\hline Ti I & & & -0.420 & -0. & 2 & +0.13 & +0.13 & +0.13 & - & +0.03 \\
\hline & & 1.067 & -1.420 & -1.4 & 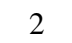 & & +0.23 & +0.33 & +0.28 & +0.08 \\
\hline & & & - & & 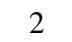 & & & & & \\
\hline $\mathrm{Ti} \mathrm{I}$ & & & - & -1 & 2 & & +0 & +0 & +0.34 & +0.03 \\
\hline Ti I & & 1.4 & - & -1 . & 2 & +0.20 & +0.20 & +0.28 & - & +0.03 \\
\hline & & 2.060 & - & & 2 & & - & +0.16 & +0.23 & +0.08 \\
\hline & & & - & & - & & & & - & - \\
\hline T1 1 & & & -1.220 & -1 & 2 & & & +0.23 & +0.26 & +0.01 \\
\hline Ti I & & 1.460 & -1.070 & -1. & 2 & +0.33 & +0.43 & +0.43 & - & +0.08 \\
\hline Ti II & & 2.050 & - & -2.480 & 2 & +036 & +0.33 & +0.40 & +0.40 & +0.28 \\
\hline & 6599.130 & 0.900 & -2.090 & -2.085 & 0 & +0.33 & +0.33 & +0.40 & - & +0.08 \\
\hline $\mathrm{Ti}$ & & 0.900 & - & -1. & 2 & & +0.28 & +0.13 & +0.03 & -0.02 \\
\hline Zr I & 6143.216 & 0.070 & -1.100 & -1.10 & 6 & -0.20 & -0.20 & 0.00 & - & -0.30 \\
\hline Ba II & 6141.728 & 0.704 & -0.080 & -0 & 8 & +0.27 & +0.27 & +0.27 & +0.57 & +0.15 \\
\hline & 6496.900 & 0.60 & -0.380 & -0.38 & 9 & +0.32 & +0.42 & +0.40 & +0.47 & +0.30 \\
\hline & 6390.483 & 0.320 & -1.450 & -1.41 & 10 & & -0.07 & +0.12 & - & +0.12 \\
\hline $\mathrm{Eu} \mathrm{II}$ & 6645.127 & 1.380 & +0.200 & +0.12 & 10 & +0.40 & +0.37 & +0.37 & +0.15 & +0.35 \\
\hline
\end{tabular}


Table 7. Mean abundance values and internal error $\sigma$ for the 5 sample stars of 47 Tucanae.

\begin{tabular}{|c|c|c|c|c|c|c|c|c|c|c|c|}
\hline \multirow[b]{2}{*}{ Species } & \multirow[b]{2}{*}{$\log \epsilon_{\odot}(\mathrm{X})$} & \multicolumn{2}{|r|}{ M 8} & \multicolumn{2}{|r|}{ M 11} & \multicolumn{2}{|r|}{ M 12} & \multicolumn{2}{|r|}{ M 21} & \multicolumn{2}{|r|}{ M 25} \\
\hline & & $\mathrm{N}$ & {$[\mathrm{X} / \mathrm{Fe}]$} & $\mathrm{N}$ & {$[\mathrm{X} / \mathrm{Fe}]$} & $\mathrm{N}$ & {$[\mathrm{X} / \mathrm{Fe}]$} & $\mathrm{N}$ & {$[\mathrm{X} / \mathrm{Fe}]$} & $\mathrm{N}$ & {$[\mathrm{X} / \mathrm{Fe}]$} \\
\hline O I & 8.77 & 2 & +0.36 & 2 & +0.22 & 2 & +0.50 & 1 & +0.31 & 2 & +0.35 \\
\hline $\mathrm{Na} \mathrm{I}$ & 6.33 & 2 & $+0.09 \pm 0.05$ & 2 & $+0.28 \pm 0.01$ & 2 & $0.01 \pm 0.04$ & 1 & -0.15 & 2 & $-0.07 \pm 0.03$ \\
\hline $\mathrm{Al} \mathrm{I}$ & 6.47 & 2 & +0.13 & 2 & +0.13 & 1 & +0.03 & $*$ & - & 2 & $+0.22 \pm 0.04$ \\
\hline Mg I & 7.58 & 2 & +0.22 & 2 & +0.22 & 2 & $+0.24 \pm 0.02$ & $*$ & - & 2 & $+0.27 \pm 0.05$ \\
\hline Si I & 7.55 & 6 & $+0.28 \pm 0.10$ & 5 & $+0.20 \pm 0.04$ & 5 & $+0.22 \pm 0.11$ & 5 & $+0.27 \pm 0.04$ & 4 & $+0.23 \pm 0.08$ \\
\hline $\mathrm{Ca} \mathrm{I}$ & 6.36 & 13 & $+0.02 \pm 0.11$ & 12 & $-0.04 \pm 0.10$ & 12 & $-0.01 \pm 0.10$ & 10 & $+0.09 \pm 0.16$ & 12 & $-0.05 \pm 0.10$ \\
\hline Ti I & 5.02 & 15 & $+0.24 \pm 0.12$ & 14 & $+0.24 \pm 0.11$ & 14 & $+0.27 \pm 0.11$ & 9 & $+0.19 \pm 0.09$ & 13 & $+0.10 \pm 0.14$ \\
\hline Ti II & 5.02 & 2 & $+0.29 \pm 0.08$ & 1 & +0.33 & 2 & $+0.28 \pm 0.17$ & 2 & $+0.31 \pm 0.11$ & 2 & $+0.18 \pm 0.14$ \\
\hline $\mathrm{ZrI}$ & 2.60 & 1 & -0.20 & 1 & -0.20 & 1 & +0.00 & - & - & 1 & -0.30 \\
\hline Ba II & 2.13 & 2 & $+0.30 \pm 0.03$ & 2 & $+0.35 \pm 0.10$ & 2 & $+0.34 \pm 0.08$ & 2 & $+0.52 \pm 0.07$ & 2 & $+0.23 \pm 0.11$ \\
\hline La II & 1.22 & 1 & +0.03 & 1 & -0.07 & 1 & +0.12 & - & - & 1 & +0.12 \\
\hline Eu II & 0.54 & 1 & +0.40 & 1 & +0.37 & 1 & +0.37 & 1 & +0.15 & 1 & +0.35 \\
\hline \multicolumn{12}{|c|}{$[\mathrm{Fe} / \mathrm{H}]$} \\
\hline $\mathrm{Fe} \mathrm{I}$ & 7.51 & 122 & $-0.62 \pm 0.11$ & 113 & $-0.62 \pm 0.11$ & 119 & $-0.63 \pm$ & 124 & $-0.77 \pm$ & 130 & $-0.64 \pm 0.11$ \\
\hline $\mathrm{Fe}$ II & 7.51 & 13 & $-0.65 \pm 0.11$ & 14 & $-0.62 \pm 0.15$ & 14 & $-0.68 \pm 0.11$ & 12 & $-0.82 \pm 0.10$ & 15 & $-0.67 \pm 0.15$ \\
\hline
\end{tabular}

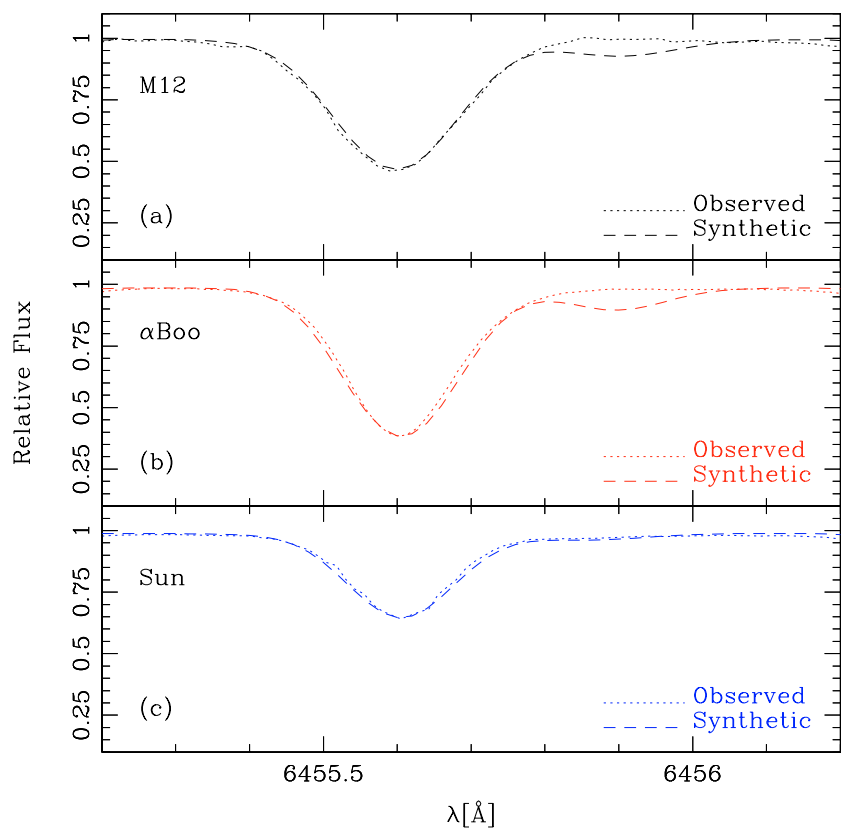

Fig. 7. Computed CaI $6455.5 \AA$ line for the solar, Arcturus and 47 Tuc: M 12 spectra, illustrating the reliability of the atomic constants.

the plane at $Z \sim 3 \mathrm{kpc}$, it belongs to neither the disk nor the bulge; on the other hand, it has a low eccentricity orbit and rotates around the Galaxy at $161 \mathrm{~km} \mathrm{~s}^{-1}$ to $191 \mathrm{~km} \mathrm{~s}^{-1}$ (Dinescu et al. 1999; Dauphole et al. 1996).

In the following we try to use the abundance ratios derived in this work as indicators of stellar population signatures. A low $[\mathrm{Ca} / \mathrm{Fe}]=0.0$ is compatible with the low $[\mathrm{Ca} / \mathrm{Fe}] \approx+0.14$ found in bulge field stars by MR94 and with $[\mathrm{Ca} / \mathrm{Fe}] \approx-0.4$ in stars of the bulge cluster NGC 6528 (Zoccali et al. 2004). A high $[\mathrm{Ba} / \mathrm{Fe}]=+0.31$ is compatible with high values of $[\mathrm{Ba} / \mathrm{Fe}]=+0.34$ and +0.60 found for $\mathrm{M} 71$ by
Table 8. Abundance uncertainties for a $\Delta T_{\text {eff }}=100 \mathrm{~K}, \Delta \log g=+0.2$, $\Delta v_{\mathrm{t}}=0.2 \mathrm{~km} \mathrm{~s}^{-1}$ and corresponding total error.

\begin{tabular}{|c|c|c|c|c|}
\hline Abundance & $\begin{array}{c}\Delta T \\
(100 \mathrm{~K}) \\
(2)\end{array}$ & $\begin{array}{c}\Delta \log g \\
(+0.2 \text { dex }) \\
\quad(3)\end{array}$ & $\begin{array}{c}\Delta v_{\mathrm{t}} \\
\left(+0.2 \mathrm{~km} \mathrm{~s}^{-1}\right) \\
(4)\end{array}$ & $\begin{array}{c}\left(\sum x^{2}\right)^{1 / 2} \\
(5)\end{array}$ \\
\hline \multicolumn{5}{|c|}{ M11 } \\
\hline$[\mathrm{Fe} / \mathrm{H}](\mathrm{I})$ & -0.02 & +0.04 & -0.09 & 0.10 \\
\hline$[\mathrm{Fe} / \mathrm{H}](\mathrm{II})$ & -0.19 & +0.13 & -0.05 & 0.23 \\
\hline$[\mathrm{O} / \mathrm{Fe}]$ & +0.01 & +0.05 & -0.01 & 0.05 \\
\hline$[\mathrm{Na} / \mathrm{Fe}]$ & +0.06 & +0.11 & -0.07 & 0.14 \\
\hline$[\mathrm{Al} / \mathrm{Fe}]$ & +0.07 & 0.00 & -0.03 & 0.08 \\
\hline$[\mathrm{Mg} / \mathrm{Fe}]$ & +0.10 & +0.14 & -0.03 & 0.17 \\
\hline$[\mathrm{Si} / \mathrm{Fe}]$ & -0.09 & +0.06 & -0.01 & 0.11 \\
\hline$[\mathrm{Ca} / \mathrm{Fe}]$ & +0.10 & +0.01 & -0.10 & 0.14 \\
\hline$[\mathrm{Ti} / \mathrm{Fe}]$ & +0.13 & +0.04 & -0.08 & 0.16 \\
\hline$[\mathrm{Zr} / \mathrm{Fe}]$ & +0.19 & +0.05 & -0.08 & 0.21 \\
\hline$[\mathrm{Ba} / \mathrm{Fe}]$ & +0.01 & +0.07 & -0.20 & 0.21 \\
\hline$[\mathrm{La} / \mathrm{Fe}]$ & +0.01 & +0.06 & -0.03 & 0.07 \\
\hline$[\mathrm{Eu} / \mathrm{Fe}]$ & -0.01 & +0.08 & -0.01 & 0.08 \\
\hline \multicolumn{5}{|c|}{ M21 } \\
\hline$[\mathrm{Fe} / \mathrm{H}](\mathrm{I})$ & +0.09 & -0.01 & -0.06 & 0.11 \\
\hline$[\mathrm{Fe} / \mathrm{H}](\mathrm{II})$ & -0.13 & +0.09 & -0.06 & 0.17 \\
\hline
\end{tabular}

Ramírez \& Cohen (2002), and for M 4 by Ivans et al. (1999), clusters that have comparably high metallicities of $[\mathrm{Fe} / \mathrm{H}]=$ -0.71 and -1.18 , respectively. Figure 9 shows the abundance pattern of 47 Tuc compared to those of M71 and M4. Therefore, in terms of $[\mathrm{Ba} / \mathrm{Fe}], 47$ Tuc behaves like other 


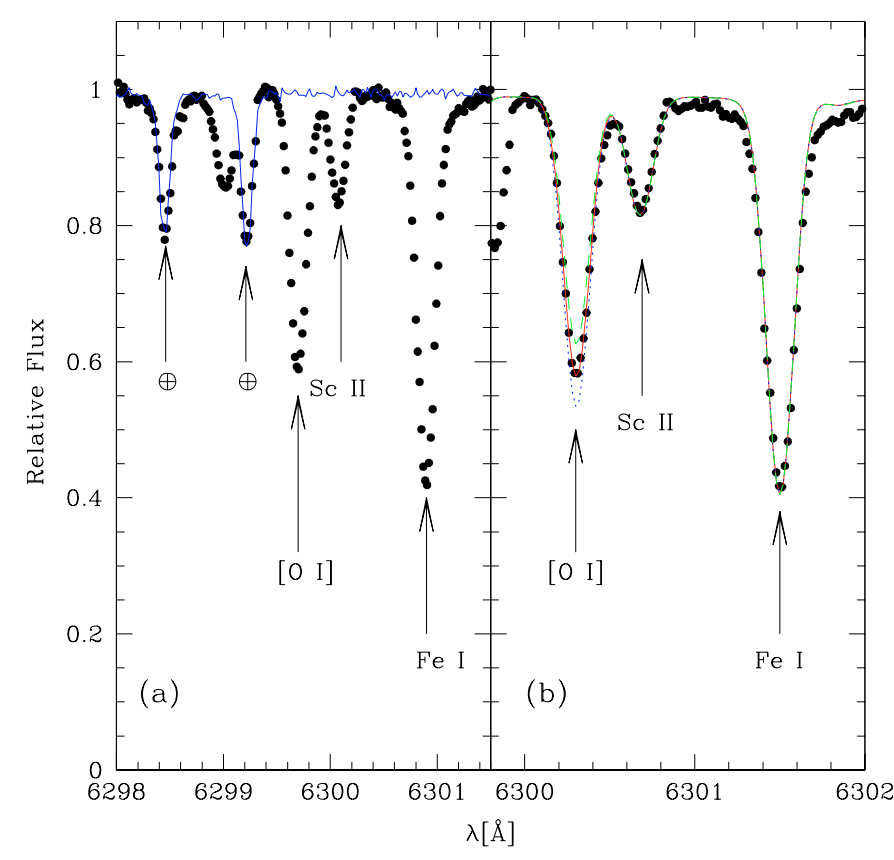

Fig. 8. a) Spectra of the reference B star HR 7316 (solid line), where the telluric lines are identified $(\oplus)$, and 47 Tuc:M12 (dotted line) showing that the [OI] line is free of blends with telluric ones. b) Observed spectrum compared to synthetic spectrum: $[\mathrm{O} / \mathrm{Fe}]=0.4$ (dashed line), 0.5 (solid line) and 0.6 (dotted line).

Table 9. Mean abundances obtained compared with results by Brown \& Wallerstein (1992), Norris \& Da Costa (1995), Carretta et al. (2004) and James et al. (2004).

\begin{tabular}{lcccc}
\hline \hline$[\mathrm{X} / \mathrm{Fe}]$ & This work & BW92 & NC95 & $\mathrm{C} 04 / \mathrm{J} 04$ \\
\hline$[\mathrm{Fe} / \mathrm{H}](\mathrm{I})$ & $-0.66 \pm 0.07$ & -0.80 & -0.72 & -0.67 \\
{$[\mathrm{Fe} / \mathrm{H}](\mathrm{II})$} & $-0.69 \pm 0.07$ & - & -0.51 & - \\
{$[\mathrm{O} / \mathrm{Fe}]$} & $+0.35 \pm 0.10$ & +0.43 & +0.17 & +0.23 \\
{$[\mathrm{Na} / \mathrm{Fe}]$} & $+0.03 \pm 0.11$ & +0.11 & +0.21 & +0.23 \\
{$[\mathrm{Al} / \mathrm{Fe}]$} & $+0.13 \pm 0.08$ & +0.67 & +0.38 & +0.23 \\
{$[\mathrm{Mg} / \mathrm{Fe}]$} & $+0.24 \pm 0.04$ & +0.49 & +0.29 & +0.40 \\
{$[\mathrm{Si} / \mathrm{Fe}]$} & $+0.24 \pm 0.02$ & +0.39 & +0.34 & +0.30 \\
{$[\mathrm{Ca} / \mathrm{Fe}]$} & $0.00 \pm 0.04$ & +0.06 & +0.16 & +0.20 \\
{$[\mathrm{TiI} / \mathrm{Fe}]$} & $+0.21 \pm 0.07$ & +0.16 & +0.27 & +0.26 \\
{$[\mathrm{TiII} / \mathrm{Fe}]$} & $+0.28 \pm 0.04$ & +0.16 & +0.56 & +0.38 \\
{$[\mathrm{Zr} / \mathrm{Fe}]$} & $-0.17 \pm 0.12$ & -0.22 & - & - \\
{$[\mathrm{Ba} / \mathrm{Fe}]$} & $+0.31 \pm 0.07$ & -0.22 & -0.04 & +0.29 \\
{$[\mathrm{La} / \mathrm{Fe}]$} & $+0.05 \pm 0.10$ & +0.24 & -0.38 & - \\
{$[\mathrm{Eu} / \mathrm{Fe}]$} & $+0.33 \pm 0.04$ & +0.36 & -0.39 & +0.14 \\
\hline
\end{tabular}

metal-rich "halo" clusters, whereas in terms of $[\mathrm{Ca} / \mathrm{Fe}]$, it behaves like bulge stars.

Despite the lack of abundances for stars with $-0.9<[\mathrm{Fe} / \mathrm{H}]<-0.65$, we present a tentative comparison of samples of field stars at a metallicity of $[\mathrm{Fe} / \mathrm{H}] \approx-0.7$, in particular those of Pompéia et al. (2003) for bulgelike stars with high velocities and eccentric orbits, and the disk samples of Chen et al. (2000), Koch \& Edvardsson (2002), Reddy et al. (2003), and Bensby et al. (2004). Figure 9 shows that $[\mathrm{O} / \mathrm{Fe}]=$ +0.35 is compatible with the thick disk values given by Bensby et al. (2004), and with the bulgelike stars, although the

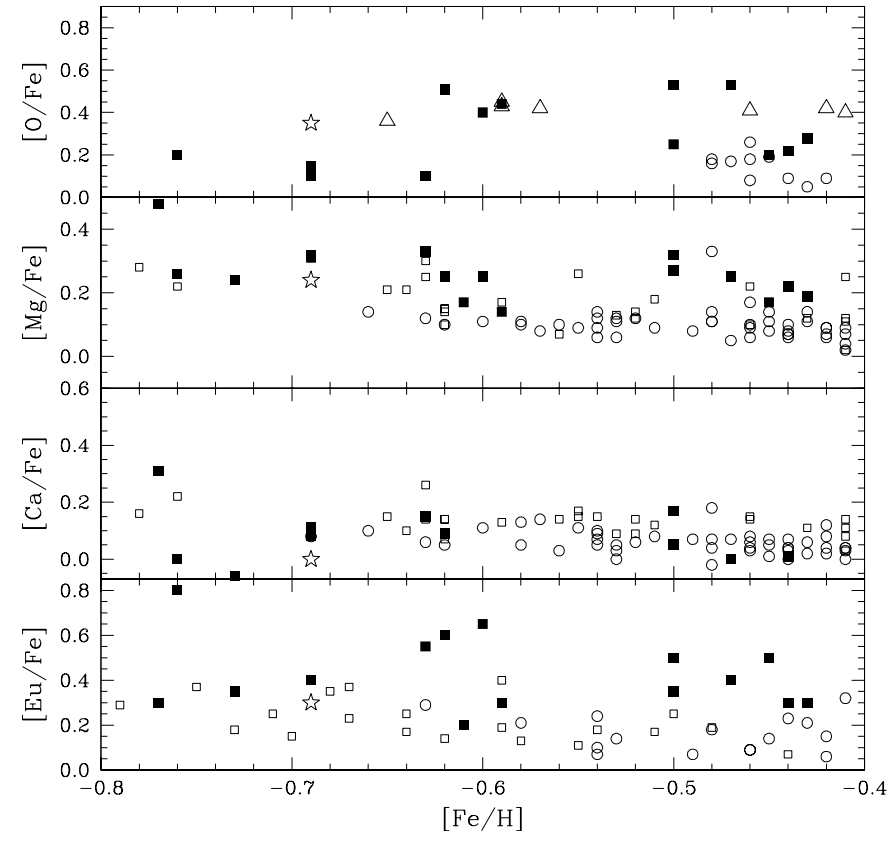

Fig. 9. a) $[\mathrm{O} / \mathrm{Fe}]$ vs. $[\mathrm{Fe} / \mathrm{H}]$, b) $[\mathrm{Mg} / \mathrm{Fe}]$ vs. $[\mathrm{Fe} / \mathrm{H}]$, c) $[\mathrm{Ca} / \mathrm{Fe}]$ vs. $[\mathrm{Fe} / \mathrm{H}], \mathbf{d})[\mathrm{Eu} / \mathrm{Fe}]$ vs. [Fe/H]: open star: mean value of $47 \mathrm{Tuc}$ (present work); filled squares: bulgelike stars by Pompéia et al. (2003); open triangles: thick disk stars by Bensby et al. (2004); open circles: disk stars by Reddy et al. (2003); open squares: disk stars by Chen et al. (2000) or Koch \& Edvardsson (2002) in the case of Eu.
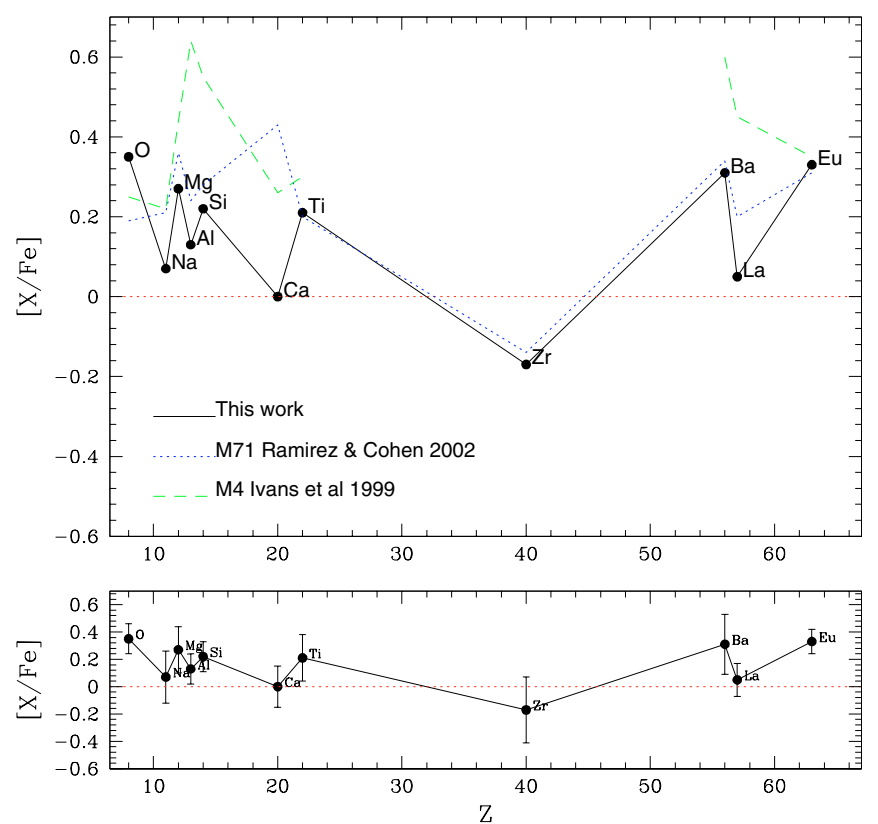

Fig. 10. Upper panel: abundance pattern of 47 Tuc compared to those of M 71 and M4; lower panel: abundance pattern with its associated error bars. The error bars quoted correspond to the quadrature of the uncertainties reported in Tables 8 and 9.

latter shows a spread of values from $[\mathrm{O} / \mathrm{Fe}]=+0.1$ to +0.5 for metallicities around $[\mathrm{Fe} / \mathrm{H}]=-0.6$ to -0.7 (Fig. 9a). $[\mathrm{Mg} / \mathrm{Fe}]=+0.24$ is compatible with both disk values in Chen et al. (2000) and Reddy et al. (2003) and with bulgelike samples (Fig. 9b). $[\mathrm{Ti} / \mathrm{Fe}]=+0.25$ is more compatible with the 
bulgelike stars, than the trend of behaviour given by Chen et al. (2000), and Reddy et al. (2003). $[\mathrm{Ca} / \mathrm{Fe}]=0.0$ is also more compatible with bulgelike stars than the higher values around $[\mathrm{Ca} / \mathrm{Fe}] \approx 0.15$ by Chen et al. (2000) and Reddy et al. (2003) (Fig. 9c). $[\mathrm{Eu} / \mathrm{Fe}]=+0.33$ is in good agreement with both the disk stars by Koch \& Edvardsson (2002) and the bulgelike stars (Fig. 9d).

Further comparison of our values with those of Reddy et al. (2003) for the thin and thick disk (their Table 11) shows that 47 Tuc is compatible with thick disk values, except for $\mathrm{Ba}$, since those authors found $[\mathrm{Ba} / \mathrm{Fe}]=0.02$ and -0.10 for thin and thick disks, respectively.

A comparison with the halo can be done by taking the mean values given in Cayrel et al. (2004). Their "plateau-like" values can be assumed as representative of the halo, since they are constant for a wide range of metallicities, except for the very low metallicities around $[\mathrm{Fe} / \mathrm{H}]<-3.5$. The halo values are $[\mathrm{O} / \mathrm{Fe}] \approx+0.5,[\mathrm{Mg} / \mathrm{Fe}] \approx+0.3,[\mathrm{Ca} / \mathrm{Fe}] \approx+0.35$, and $[\mathrm{Ti} / \mathrm{Fe}] \approx+0.25$, indicating that 47 Tuc also has abundances typical of halo stars, except for its low Ca-to-Fe.

Comparisons with disk, thick disk, bulgelike samples, bulge, and halo abundances do not indicate a clear preferential membership of 47 Tuc to any of these populations. It is more compatible with the halo values, but $\mathrm{Ca}$ is low; $\mathrm{Ca}$ is low in the bulge, but there $\mathrm{Ba}$ is also low, unlike 47 Tuc. Another possibility is that 47 Tuc has been formed in a dwarf galaxy that merged with the Galaxy in its early phases, scenario proposed e.g. by Burstein et al. (2004).

\section{Conclusions}

We addressed the issue of abundance ratios in 47 Tucanae, using high resolution and high signal-to-noise spectra obtained with the UVES spectrograph at the Kueyen VLT-UT2 telescope. We find $[\mathrm{O} / \mathrm{Fe}]=+0.35,[\mathrm{Ca} / \mathrm{Fe}]=0.0$, a mean value of $[\mathrm{Mg}, \mathrm{Si}, \mathrm{Ti} / \mathrm{Fe}]=+0.23$ and $[\mathrm{Na}, \mathrm{Al} / \mathrm{Fe}]=+0.1,[\mathrm{La} / \mathrm{Fe}]=0.05$, $[\mathrm{Zr} / \mathrm{Fe}]=-0.17,[\mathrm{Ba} / \mathrm{Fe}]=+0.31$ and $[\mathrm{Eu} / \mathrm{Fe}]=0.33$. An overall metallicity $[\mathrm{M} / \mathrm{H}]=-0.45$ and $Z=0.006$ is obtained, with $Z$ the parameter needed for comparison with isochrones, where we assumed all $\alpha$-elements (including the non-observed ones $\mathrm{S}, \mathrm{Ar}, \mathrm{Ne}$ ) to be enhanced by $0.3 \mathrm{dex}$.

Most abundance characteristics resemble those of other metal-rich halo clusters, in particular M 4 and M 71, two wellstudied globular clusters with similar metallicities, except for the low Ca-to-Fe in 47 Tuc. The $\alpha$ elements (including the low $\mathrm{Ca}$ ) and $\mathrm{Eu}$ are compatible with bulge globulars, field bulgelike and thick disk stars, except for the high Ba-to-Fe. Therefore, from abundance ratios, as well as from kinematical indicators, 47 Tuc does not appear to be either a typical disk or a bulge or even a halo cluster. From the O-to-Fe ratio it could be a thick disk member, but very few other abundances are available to offer reliable thick disk samples. Clearly, further investigations are needed in two directions: abundances (i) in field stars with $-0.9<[\mathrm{Fe} / \mathrm{H}]<-0.65$, and particularly those with the kinematics of thick disk, and (ii) in globular clusters with derivation of a full range of elements.

Acknowledgements. A.A. acknowledges a Fapesp fellowship No. 2002/02668-4. B.B. acknowledges grants from CNPq and
Fapesp. DM acknowledges grants from FONDAP Center for Astrophysics 15010003 . This publication makes use of data products from the Two Micron All Sky Survey, which is a joint project of the University of Massachusetts and the Infrared Processing and Analysis Center/California Institute of Technology, funded by the National Aeronautics and Space Administration and the National Science Foundation.

\section{Appendix A: Iron line list}

The Fe I and Fe II lines employed for the analysis are given in Table A.1, available only in electronic form at the CDS via anonymous ftp 130.79.128.5.

\section{References}

Allende Prieto, C., Lambert, D. L., \& Asplund, M. 2001, ApJ, 556, L63

Alonso, A., Arribas, S., \& Martínez-Roger, C. 1999, A\&AS, 140, 261 (AAM99)

Alonso, A., Arribas, S., \& Martínez-Roger, C. 2001, A\&A, 376, 1039

Armandroff, T. E. 1989, AJ, 97, 375

Ballester, P., Modigliani, A., Boitquin, O., et al. 2000, in The Messenger, 101, 31

Barbuy, B., Bica, E., \& Ortolani, S. 1998, A\&A, 333, 117

Barbuy, B., Renzini, A., Ortolani, S., Bica, E., \& Guarnieri, M. D. 1999a, A\&A, 341, 539

Barbuy, B., Ortolani, S., Bica, E., \& Desidera, S. 1999b, A\&A, 348, 783

Barbuy, B., Perrin, M.-N., Katz, D., et al. 2003, A\&A, 404, 661

Barklem, P. S., Anstee, S. D., \& O’Mara, B. J. 1998, PASA, 15, 336

Barklem, P. S., Piskunov, N. E., \& O'Mara, B. J. 2000, A\&AS, 142, 467

Bell, R.A., Eriksson, K., Gustafsson, B., \& Nordlund, Å. 1976, A\&AS, 23, 37

Bensby, T., Feltzing, S., \& Lundström, I. 2003, A\&A, 410, 527

Bensby, T., Feltzing, S., \& Lundström, I. 2004, A\&A, 415, 155

Biehl, D. 1976, Ph.D. Thesis, University of Kiel

Brown, J. A., Wallerstein, G., \& Oke, J. B. 1990, AJ, 100, 1561

Brown, J. A., \& Wallerstein, G. 1992, AJ, 104, 1818 (BW92)

Burstein, D., Li, Y., Freeman, K., et al. 2004, ApJ, 614, 158

Carretta, E., Cohen, J. G., Gratton, R. G., \& Behr, B. B. 2001, AJ, 122 , 1469

Carretta, E., Gratton, R.G., Bragaglia, A., Bonifacio, P., \& Pasquini, L. 2004, A\&A, 416, 925 (C04)

Cayrel, R. 1988, in The Impact of Very High S/N Spectroscopy on Stellar Physics, G. Cayrel de Strobel, \& M. Spite (Dordrecht: Kluwer), IAU Symp., 132, 345

Cayrel, R., Perrin, M.-N., Barbuy, B., \& Buser, R. 1991, A\&A, 247, 108

Cayrel, R., Depagne, E., Spite, M., et al. 2004, A\&A, 516, 1117

Chen, Y. Q., Nissen, P. E., Zhao, G., Zhang, H. W., \& Benoni, T. 2000, A\&AS, 141, 491

Coelho, P., Barbuy, B., Schiavon, R. P., Meléndez, J., \& Castilho, B. V. 2005, A\&A, in preparation

Cohen, J. G., Gratton, R. G., Behr, B., \& Carretta, E. 1999, ApJ, 523, 739

Côté, P. 1999, AJ, 118, 406

Cram, L. 1999, Trans. IAU XXIIIB, ed. J. Andersen, p. 141

Davies, R. L., Sadler, E. M., \& Peletier, R. F. 1993, MNRAS, 262, 650

Dean, J. F., Warpen, P. R., \& Cousins, A. J. 1978, MNRAS, 183, 569

Dauphole, B., Geffert, M., Colin, J., et al. 1996, A\&A, 313, 119 
Dinescu, D. T., Girard, T. M., \& van Altena, W. F. 1999, AJ, 117, 1792

Edvardsson, B., Andersen, J., Gustafsson, B., et al. 1993, A\&A, 102, 603

Frenk, C. S., \& White, S. D. M. 1982, MNRAS, 198, 173

Geisler, D., Grebel, E. K., \& Minniti, D. 2002, IAU Symp., 207, Extragalactic Star Clusters, ASP, USA

Gratton, R. G., Carretta, E., Eriksson, K., \& Gustafsson, B. 1999, A\&A, 350, 955

Grevesse, N.,\& Sauval, J. 1998, SSRev, 35, 161

Gustafsson, B., Bell, R. A., Eriksson, K., \& Nordlund, Å. 1975, A\&A, 42, 407

Harris, W. E. 1996, AJ, 112, 1487

Hinkle, K., Wallace, L., Valenti, J., \& Harmer, D. 2000, Visible and Near Infrared Atlas of te Arcturus spectrum 3727-9300 (San Francisco: ASP)

Houdashelt, M. L., Bell, R. A., \& Sweigart, A. V. 2000, AJ, 119, 1448

Ivans, I., Sneden, C., Kraft, R. P., et al. 1999, AJ, 118, 1273

James, G., François, P., Bonifacio, P., et al. 2004, A\&A, 427, 825

Kim, Y., Demarque, P., Yi, S. K., \& Alexander, D. R. 2002, ApJS, 143, 499

Koch, A., \& Edvardsson, B. 2002, A\&A, 381, 500

Kraft, R. P. 1998, Highlights Astron., 11, 53

Landolt, A. U. 1992, AJ, 104, 372

Lawler J. E., Bonvallet G., \& Sneden, C. 2001a, ApJ, 556, 452.

Lawler J. E., Wickliffe M.E., Hartog A.D. 2001b, ApJ, 563, 1075

Martin, W. C., Fuhr, J. R., Kelleher, D. E., et al. 2002, NIST Atomic Database (version 2.0), http://physics.nist.gov/asd National Institute of Standards and Technology, Gaithersburg, MD

McWilliam, A. 1998, AJ, 115, 1640

McWilliam, A., \& Rich, R. M. 1994, ApJS, 91, 749 (MR94)
Meléndez, J., Barbuy, B., Bica, E., et al. 2003, A\&A, 411, 417

Meléndez, J., \& Barbuy, B. 2005, A\&A, in preparation

Menzies, J. 1973, MNRAS, 163, 323

Minniti, D. 1995a, A\&AS, 113, 299

Minniti, D. 1995b, AJ, 109, 1663

Momany, Y., Piotto, G., Recio-Blanco, A., et al. 2002, ApJ, 576, L65

Norris, J. E., \& Da Costa, G. S. 1995, ApJ, 447, 680

Origlia, L., Rich, R. M., \& Castro, S. 2002, AJ, 123, 1559

Plez, B., Brett, J. M., \& Nordlund, A.. 1992, A\&A, 256, 551

Pompéia, L., Barbuy, B., \& Grenon, M. 2003, ApJ, 592, 1173

Ramírez, S. V., \& Cohen, J. G. 2002, AJ, 123, 3277

Reddy, B. E., Tomkin, J., Lambert, D. L., \& Allende Prieto, C. 2003, MNRAS, 340, 304

Rieke, G. H., \& Lebofsky, M. J. 1985, ApJ, 288, 618

Rutledge, G. A., Hesser, J. E., Stetson, P. B., et al. 1997, PASP, 109, 883

Rutten, R. J. 1978, SoPh, 56, 237

Skrutskie, M., Schneider, S.E., Stiening, R., et al. 1997, in The Impact of Large Scale Near-IR Sky Surveys, ed. Garzon et al. (Netherlands: Kluwer), 210, 187

Spite, M. 1967, Ann. Astroph., 30, 211

Stetson, P. B. 1994, PASP, 106, 250

Stetson, P. B., \& Pancino, E. 2005, in preparation

Takeda, Y., Zhao, G., Takada-Hidai, M., et al. 2003, Chin. J. Astron. Astroph., 3, 316

Valdes, F. G. 1998, ASP Conf. Ser. 145: Astronomical Data Analysis Software and Systems VII, 7, 53

Zinn, R. 1985, ApJ, 293, 424

Zoccali, M., Renzini, A., Ortolani, S., et al. 2001, ApJ, 553, 733

Zoccali, M., Barbuy, B., Hill, V., et al. 2004, A\&A, 423, 507 